\title{
Clinical scope of early fetal echocardiography
}

\author{
Ximena Carolina Romero Infante ${ }^{1}$, Arturo Montaño Mendoza ${ }^{1}$, Diana Sarmiento ${ }^{1}$, María \\ Uriel Calvo ${ }^{1}$, and José De la Hoz Valle ${ }^{1}$ \\ ${ }^{1}$ Universidad El Bosque Facultad de Medicina
}

February 17, 2021

\begin{abstract}
The chances of detecting congenital heart disease are improved following structured protocols. Fetal heart disease is one of the main serious congenital malformations. The objective of this review is to present the benefits of sequential and routine cardiac evaluation in the first and early second trimesters and to identify the structures and pathologies that can be detected at this gestational age. The databases of PubMed, Medline, MD consult, Embase, Clinical Key, Scielo, and ScienceDirect, as well as specialized texts in Spanish and English were searched for diagnostic studies, systematic reviews, and meta-analysis related to the terms "early fetal echocardiography" and "congenital heart malformations" published between 2000 and 2019 . Technological advances have revolutionized the fetal echocardiographic examination making possible the diagnosis of congenital heart disease from the first and early second trimester of pregnancy. However, it should be recognized that early fetal echocardiography has limitations such as the evaluation of pulmonary veins and cardiac lesions that are progressive. The benefit found was earlier detection of pathologies with high sensitivity and specificity. A fetal cardiac evaluation sequence was included in these early fetal echocardiographic examinations due to the awareness of fetal anatomical and hemodynamic differences in the first trimester and the beginning of the second trimester. Early fetal echocardiography is a very useful tool for the detection of congenital heart disease; it allows parents to be informed about the prognosis and possible treatments early. Fetal cardiac examination in the first trimester is safe and can detect the most of heart congenital malformation.
\end{abstract}

\section{INTRODUCTION}

Congenital heart disease diagnosed in the first trimester was described in the early 1990s using transvaginal ultrasound. Subsequently, the value of transabdominal ultrasound was demonstrated through technological advances and revolutionized fetal echocardiographic examination, making it possible to diagnose congenital heart defects from the first trimester and very early in the second trimester of pregnancy $(1,2)$.

Fetal heart diseases are one of the main serious congenital malformations, affecting approximately 0.8 to $1 \%$ of newborns. It is the most frequent fetal anomaly, occurring three to four times more than neural tube malformations and five to seven times more than chromosomal abnormalities (1).

Additionally, these pathologies are responsible for more than $50 \%$ of infant mortality due to congenital anomalies and 20-30\% of neonatal deaths; therefore, it is important to diagnose and understand the nature of the pathology early, as well as anticipate results to offer parents management plans and optional treatments that could include the possibility of termination of pregnancy $(1,3)$.

The objective of this review is to present the benefits of sequential and routine cardiac evaluation in the first and early second trimester and to identify the structures and pathologies that can be detected at this gestational age.

\section{MATERIALS AND METHODS}

A systematized review of the literature was performed using PubMed, Medline, MD Consult, Ovid, Embase, Clinical Key, Scielo, and ScienceDirect databases. The search included studies of diagnostic tests, systematic 
reviews, meta-analyses with the combination of terms "Pregnancy Trimester, First", "Fetal Heart", "Ultrasonography, Prenatal", "Heart Defects, Congenital", and "Echocardiography". Documents published in the period from 2000 to 2019 were included in the search. A predetermined search strategy was used to identify qualitative and quantitative studies that included echocardiography in the first trimester and early second trimester.

The selection of the studies was carried out using the Prisma methodology $(4,5)$ by collecting the articles, reading titles and abstracts, organizing them in an Excel table, and identifying the characteristics of the articles, such as year of publication, design, number of patients, institution, objective, years of collection of the participants, findings from cardiac echocardiography, and conclusions, among other parameters.

Early fetal cardiac physiology, detection rates, possible cardiac pathologies in the first trimester, clinical indications, techniques, safety, and sequencing of cardiac imaging, and the strengths and weaknesses of early fetal echocardiography were reviewed.

\section{RESULTS AND DISCUSSION}

In the databases of PubMed, Medline, MD Consult, Ovid, Embase, Clinical Key, Scielo, and ScienceDirect, 47 articles with the characteristics of eligibility were found. After eliminating duplicate articles, the inclusion and exclusion criteria, such as studies with low epidemiological rigor, opinion articles, unacceptable study populations, among others, were applied. A total of 22 articles were finally selected for review (Figure 1).

The results and discussion were organized according to the research questions asked to reach the main objective in the studies. The questions that were raised answer the objective.

\section{What is the detection rate of cardiac pathologies diagnosed early?}

Studies were found in the systematized review of the literature $(6,7)$, which included a large series. One of the largest was performed by Khalil et al. (7) and included a little more than 44,000 single pregnancies where they performed the first-trimester ultrasound as part of routine examinations for aneuploidies, evidencing a detection rate of the main congenital heart defects in up to $34 \%$ of the patients.

Another multicenter study included more than 1000 patients, and it was concluded that the detection rate of major heart diseases between weeks 11 and 13 of gestation was $29 \%$ (2,7-11). Among the early detectable heart diseases are those shown in table I.

Other cardiac alterations may not be identified in the first and early second trimesters, such as those indicated in table II.

In the different systematic reviews on early fetal echocardiography, it was shown that the first trimester examinations diagnosed important congenital heart diseases with high precision, with a sensitivity of $85 \%$ and a specificity of $97 \%(8,9,12)$. Therefore, successful effective ultrasound evaluation of the fetal heart early could permit to interfere significantly with the viability of a pregnancy.

\section{What are the indications for early echocardiography?}

There are maternal and fetal indications. Within the maternal, there is the family history of heart disease, diabetes mellitus, autoimmune diseases, pregnancies conceived by assisted reproduction by both in vitro fertilization (IVF) alone and IVF with intra-cytoplasmic sperm injection (ICSI), exposure to teratogenic drugs, among others $(1,6,11,13)$.

Among the fetal indications, there are monochorionic twin pregnancies; the presence of extracardiac malformations; and ultrasound findings, such as nuchal translucency (NT) equal to or greater than $3.5 \mathrm{~mm}$ or above P95 for gestational age. These ultrasound findings imply the need to perform some type of invasive test (chorionic villus biopsy or amniocentesis) to establish the fetal karyotype, and thus, determine the presence of chromosomal abnormalities $(1,3,8,9)$. 
However, increased NT in the presence of a normal karyotype is associated with congenital heart disease by up to $44 \%$ and this risk makes the importance of early echocardiography greater $(1,9,14,15)$.

The sensitivity of increased NT as a screening tool for major cardiac diseases is increased if it is related to other markers of cardiac function, such as a venous ductus (DV) with an absent or reverse A wave as well as an index of pulsatility (IP) $>95$ (Figure 2), and/or tricuspid regurgitation (RT) (Figure 3) $(2,9,10,12,14$ ).

The presence of a NT level [?] $3.5 \mathrm{~mm}$ triples the risk of congenital heart defects $(1,9,12,14,15)$. However, finding a DV with a normal flow pattern halves the risk of heart disease $(2,9,10,16)$. Current meta-analyses have concluded that normal NT levels and a DV with abnormal A wave or IP> 95 is associated with $19 \%$ of heart diseases with a false positive rate of $4 \%$ while an increase in NT levels associated with a DV with abnormal A wave or IP> 95 is associated with up to $73 \%$ and $55 \%$ of congenital heart disease, respectively, with a false positive rate of $20 \%(2,10,12,16)$.

Regarding the RT, it was frequently seen in fetuses with trisomy on ultrasound at weeks 11 to 14 although it can also be identified in euploid fetuses with congenital heart defects. Its presence increases the risk of congenital heart defects up to 8 times when an increased NT coexists $(8,10,12,17)$.

Any of the three mentioned ultrasound markers (increased NT, altered DV, and RT) can be found in up to $57.6 \%$ of fetuses with congenital heart disease and $8 \%$ of fetuses with normal hearts $(8,10,12,14,16,17)$.

It can be concluded that a DV with an IP> 95 and/or an abnormal A wave in the first trimester are associated with congenital heart defects by $64 \%$ on average when the NT level is abnormal. This is also reproducible for RT, likewise, the association rate is significantly reduced if these findings are absent $(2,9,10,12,16,17)$.

\section{What are the techniques for early fetal echocardiography?}

The most common fetal heart screening procedure nowadays is two-dimensional (2D) echocardiography $(9,18)$. Likewise, Doppler ultrasound is another instrument with which you can evaluate fetal heartbeats, visualize the direction of blood flow, assess the different heart chambers, as well as analyze the flow wave and measure its speed $(1,6,8,9,17,18)$.

To perform early fetal echocardiography, the highest available rate probes should be used; for transabdominal evaluation, $9 \mathrm{MHz}$ linear transducers or $7 \mathrm{MHz}$ convex transducers, and/or a 9-12 $\mathrm{MHz}$ probe can be used in the transvaginal examination $(1,13,17,19)$.

Transabdominal ultrasound is the most widely used method for conducting fetal echocardiography; transvaginal ultrasound is only used when visualization through the maternal abdomen is not useful to observe fetuses in the first trimester of pregnancy. The mobilization of the probe is greater through the transabdominal route; this allows multiple planes of the fetal heart to be viewed, which is more limited transvaginal $(1,6,19)$.

In mothers with obesity, it is more difficult to detect fetal heart disease at an early gestational age, which leads to expanding the ultrasound study, particularly by using transvaginal ultrasound $(1,6)$.

Restrictions of a transvaginal examination include limited angulation and the distance of the fetal heart from the cervix, making cardiac examination difficult. However, these restrictions are sometimes compensated by fetal movements. After 14 weeks of gestation, the transvaginal examination is generally not superior to the transabdominal examination $(1,6)$.

In the last three decades, new technological advances have been implemented (three-dimensional echo, 4dimensional ultrasound, tissue Doppler, STIC, Fetal Intelligent Navigation Echocardiography (FINE), Superb Microvascular Imaging (SMI) and others), complementing fetal cardiac evaluation. However, 2D ultrasound, color, and pulsed Doppler are still the fundamental tools of the fetal cardiac study. $(17,20-22)$.

\section{How safe is early fetal echocardiography?}

According to studies and based on the existing ultrasound techniques, performing multiple ultrasound examinations of fetuses over 10 weeks' gestation is not harmful. Therefore, it is a safe and effective diagnostic 
method if ultrasound safety guidelines are followed $(6,13,19)$.

Safety concerns in echocardiography are the risk of thermal injury and cavitation, this can occur when examinations are carried out at very early stages. However, these have passed into the background since the current recommendation is to keep the thermal index below 1 ideally below 0.7 and with a minimum necessary Doppler exposure. In these conditions, early fetal echocardiography is reasonably safe. $(1,9,19,25)$.

\section{What are the characteristics of the heart in early pregnancy?}

Several structural and hemodynamic aspects must be considered when studying the heart in the first trimester and early second trimester. Among them, we have symmetrical ventricular sizes, where minor discrepancies of the same can be indicative of pathologies, such as coarctation of the aorta $(1,8)$. In later stages of gestation, there may be slight asymmetries in the ventricular cavities, which can be considered as non-pathological if it is an isolated finding (7).

The great arteries should be almost symmetrical with the pulmonary trunk being slightly larger than the aorta. The difference in the size of the great arteries may indicate valve abnormalities or coarctation of the aorta, among other issues. Furthermore, the absence of one of the great vessels, transposition of them, or abnormal flows to the color Doppler evaluation could raise the possibility of major heart disease (1,7-9).

The cardiac axis is located on the midline in the first weeks of gestation with an average angle of $15^{\circ}$ to $25^{\circ}$. At around 12 weeks, its location varies to the left of the chest with an angle of around $45^{\circ}$ (Figure 4 ). On the other hand, the displacement of the atrioventricular valves can be subtle before 12 weeks of gestation, making it necessary to evaluate the short axis of the valves to rule out defects of the ventricular atrial septum. This can be viewed by rotating the transducer $90^{\circ}$ from the long axis planes (cut where the exit of the aorta between the atria and ventricles is evident), allowing the visualization of a cross-section of the ventricular cavities and the atrioventricular valves $(9,11)$.

There are other findings less relevant at this gestational age, but equally important to mention. These include the presence of a pericardial effusion between 8 and 9 weeks of gestation and the absence of a usual cardiothoracic relationship, which is altered by a larger heart size in proportion to the thorax at this gestational age. Usually, in the second and third trimester, this ratio is 0.45 or when the heart occupies a third of the fetal chest $(1,6,11,23)$.

Regarding the hemodynamic aspects, the fetal heart presents several modifications between 8 and 12 weeks of gestation. Fetal heart rate is slow at 6 weeks' gestation with an approximate value between 100 and 130 bpm (beats per minute), increasing to $160 \mathrm{bpm}$ around 9 weeks, with its maximum peak at 10 weeks of 175 $\mathrm{bpm}$, from which it slowly decreases to a value of $150 \mathrm{bpm}$ starting the second trimester. In the evaluation of first-trimester aneuploidy screening, we can find tachycardia, being more common in trisomy 13 and 21 and monosomy X, up to $180 \mathrm{bpm}$. Similarly, the presence of bradycardia below $110 \mathrm{bpm}$ could be associated with trisomy 18 and triploidy $(1,9,22)$.

Changes in ventricular filling patterns reflect relaxation or diastole. At 8 weeks gestation, there is a very short period of ventricular filling with a monophasic model, and then at 10 weeks an $\mathrm{E}$ wave gradually emerges with ventricular filling in response to increases in the cardiac cycle $(1,22)$ (Figure 5).

Changes in atrial pressure are reflected in the flow patterns of systemic veins at very early stages. There is a high wave velocity in the inferior vena cava, which is why in some cases it is normal to find the reverse A wave in the venous ductus. You can also see the pulsatile flow from the umbilical vein and the absence of flow at the end of diastole in the umbilical artery in normal findings before the tenth week of gestation (1).

\section{What sequence and types of cuts can be performed on an early fetal echocardiogram?}

For the analysis and diagnosis of congenital heart defects, it is necessary to study the fetal heart by obtaining ultrasound cuts at different levels and various angulations. Currently, the fetal echocardiographic examination is standardized and there are international protocols that outline a detailed ultrasound study to detect fetal heart disease. 
The transverse plane is fundamental to identify heart disease. In up to $98 \%$ of cases, it allows visualization of the visceral situs, situs solitus, cardiac axis and cardiothoracic relationship; the evaluation of four-chamber view $(4 \mathrm{C})$ in $100 \%$ of cases; and the Three-vessel-trachea view (3V-T) in 60-70\%. Furthermore, it improves the identification of functional pathology with the direction of flows $(8,9,11,20,23,24)$.

The sagittal plane allows assessment in 94 to $96 \%$ of cases of the aortic and ductal arches. It also allows visualization of the axis of the cavas as well as a view of the short axis of the ventricles and atrioventricular valves. Pulsed and color Doppler evaluation should be included in the study protocol for ventricular entrances, exit tracts, aortic and ductal arches, systemic veins, and umbilical vessels $(1,7,9,11,12,20)$.

Pulmonary vein evaluation is the most challenging aspect of fetal echocardiography to evaluate in early pregnancy, with a success rate of 25 to $55 \%$ even in 13 weeks of gestation $(1,11,20,23)$.

The echocardiography assessment protocol must include the aspects described in Table III.

When used by professionals with advanced knowledge, the diagnostic performance of congenital heart defects by this method has $85 \%$ sensitivity and $97 \%$ specificity for prenatal detection with a high negative predictive value and precision in detecting a significant structural defect on ultrasound scans from weeks 11 to 14 of gestation $(8,20,22)$.

\section{Novel and relevant aspects of this study}

Early fetal echocardiography is a good tool for the early detection of congenital heart defects, with a sensitivity of $85 \%$ and a specificity of $97 \%$ if performed by trained personnel using high-resolution ultrasound equipment that contains the appropriate translators as indicated by the chosen route of analysis, either abdominal and / or transvaginal $(1,8,12,19)$.

It is reasonably safe at an early age since the thermal below 1, ideally below 0.7 and with a minimum necessary Doppler exposure. Also, it reduces the probability of cavitation and thermal injuries in the fetus $(1,6,13,19,25)$.

Early echocardiography with indications, either maternal or fetal, has a high diagnostic capacity for pathologies such as tricuspid, mitral, aortic and pulmonary atresia, hypoplastic left heart syndrome, transposition of the great vessels, double outflow tract of the right ventricle, common arterial trunk, tetralogy of Fallot, atrial and ventricular septal defects. However, it limited in its ability to identify evolutionary lesions and the pulmonary venous return $(1,6,8,9,11)$.

The diagnosis of an early congenital cardiac malformation allows offering parents the most exhaustive prognosis and monitoring of the fetus. It also enables them to plan more appropriately for birth and neonatal management, as well as opening the possibility of voluntary termination of the gestation.

\section{CONCLUSIONS}

Early fetal echocardiography has become a tool for detecting congenital heart disease. There are several markers available to identify a fetus at high-risk for heart disease in the first trimester of pregnancy, including increased TN levels, absent or reverse flow of DV and the presence of RT $(2,8-10,12,14,16)$.

The fetal cardiac examination in the first trimester is reasonably safe but requires knowledge of the technical aspects and normal fetal hemodynamic characteristics when performing it at this stage of pregnancy $(1,6,11,19)$.

The experience and training of professionals are important factors related to the successful identification of fetal heart defects. In low-risk women, well-trained professionals can achieve a 40-50\% detection rate of congenital heart disease while for high-risk women, this detection rate can increase to more than $80 \%$ $(8,9,11,13)$.

By following structured protocols and guidelines for the evaluation of fetuses, using transvaginal ultrasound (if necessary), and employing new technologies, early fetal echocardiography improves the detection rate of 
congenital heart defects. Furthermore, the limitations of early fetal echocardiography should be recognized and consideration should be given to continuing standardized cardiac examinations between weeks 18 and 22 to complement the anatomical evaluation and evolution of previously identified findings.

\section{ACKNOWLEDGMENTS}

This study was conducted with the support of El Bosque Research Group of Maternal Fetal Medicine and Gynecology, Ecodiagnóstico El Bosque S. A. S, and Universidad El Bosque. Bogotá. Colombia.

\section{Author contributions}

All authors contributed to the study conception and design. Material preparation, data collection and analysis were performed by Romero Ximena, Montaño Arturo, Sarmiento Diana, Uriel Monserrat and De la Hoz José. The first draft of the manuscript was written by Romero Ximena, Montaño Arturo, Sarmiento Diana, Uriel Monserrat and De la Hoz José, and all authors commented on previous versions of the manuscript. All authors read and approved the final manuscript.

\section{REFERENCES}

1. Mcbrien A, Hornberger L. Early fetal echocardiography. Birth Defects Res. 2019;111(8):370-9.

2. Timmerman E, Clur SA, Pajkrt E, Bilardo CM. First-trimester measurement of the ductus venosus pulsatility index and the prediction of congenital heart defects. Ultrasound Obstet Gynecol. 2010;36(6):66875 .

3. Mogra R, Saaid R, Kesby G, Hayward J, Malkoun J, Hyett J. Early fetal echocardiography : Experience of a tertiary diagnostic service. Aust New Zeal J Obstet Gynaecol. 2015;55(6):552-8.

4. Thabane L, Thomas T, Ye C, Paul J. Posing the research question: Not so simple. Can J Anesth. 2009;56(1):71-9.

5. Ramirez R, Meneses J, Flores M. Una propuesta metodologica para la conduccion de revisiones sistematicas de la literatura en la investigacion biomedica. Rev Ces Mov y Salud [Internet]. 2013;1(1):61-73.

6. Carvalho JS. Fetal heart scanning in the first trimester. Prenat Diagn. 2004;24(13):1060-7.

7. Khalil A, Nicolaides KH. Seminars in Fetal \& Neonatal Medicine Fetal heart defects : Potential and pitfalls of first-trimester detection. Semin Fetal Neonatal Med [Internet]. 2013;18(5):251-60.

8. Zidere V, Bellsham-Revell H, Persico N, Allan LD. Comparison of echocardiographic findings in fetuses at less than 15 weeks' gestation with later cardiac evaluation. Ultrasound Obstet Gynecol. 2013;42(6):679-86.

9. Zidere V. First Trimester Fetal Echocardiography. In: Simpson JM, Zidere V, Miller OI, editors. Fetal Cardiology: A Practical Approach to Diagnosis and Management. 1st ed. London: Springer International Publishing AG; 2018. p. 57-70.

10. Minnella GP, Crupano FM, Syngelaki A, Zidere V, Akolekar R, Nicolaides KH. Diagnosis of major heart defects by routine first-trimester ultrasound examination: association with high nuchal translucency, tricuspid regurgitation and abnormal flow in the ductus venosus. Ultrasound Obstet Gynecol. 2020;55(5):637-44.

11. Hernandez-Andrade E, Patwardhan M, Cruz-Lemini M, Luewan S. Early Evaluation of the Fetal Heart. Fetal Diagn Ther. 2017;42(3):161-73.

12. Clur SB, Bilardo CM. Early detection of fetal cardiac abnormalities : how effective is it and how should we manage these patients? Prenat Diagn. 2014;34(13):1235-45.

13. Donofrio M, Moon-Grady A, Hornberger L, Copel J, Sklansky M, Abuhamad A, et al. Diagnosis and treatment of fetal cardiac disease: A scientific statement from the American Heart Association. Circulation. 2014;129(21):2183-242. 
14. Vogel M, Sharland GK, Allan LD, Zidere V, Simpson JM, Miller OI, et al. Prevalence of increased nuchal translucency in fetuses with congenital cardiac disease and a normal karyotype. Cardiol Young. 2009;19(5):441-5.

15. Atzei A, Gajewska K, Huggon IC, Allan L, Nicolaides KH. Relationship between nuchal translucency thickness and prevalence of major cardiac defects in fetuses with normal karyotype. Ultrasound Obstet Gynecol. 2005;26(2):154-7.

16. Papatheodorou SI, Evangelou E, Makrydimas G, Ioannidis JPA. First-trimester ductus venosus screening for cardiac defects: A meta-analysis. BJOG An Int J Obstet Gynaecol. 2011;118(12):1438-45.

17. Persico N, Moratalla J, Lombardi CM, Zidere V, Allan L, Nicolaides KH. Fetal echocardiography at 11-13 weeks by transabdominal high-frequency ultrasound. Ultrasound Obstet Gynecol. 2011;37(3):296-301.

18. Lee W, Allan L, Carvalho JS, Chaoui R, Copel J, Devore G, et al. ISUOG consensus statement : what constitutes a fetal echocardiogram ? Ultrasound Obs Gynecol . 2008;32(2):239-42.

19. Hutchinson D, Mcbrien A, Howley L, Yamamoto Y, Sekar P, Motan T, et al. First-Trimester Fetal Echocardiography : Identification of Cardiac Structures for Screening from 6 to 13 Weeks ' Gestational Age. J Am Soc Echocardiogr [Internet]. 2017;30(8):763-72.

20. Garcia M, Yeo L, Romero R, Haggerty D, Giardina I, Hassan SS, et al. Prospective evaluation of the fetal heart using Fetal Intelligent Navigation Echocardiography (FINE). Ultrasound Obstet Gynecol. 2016;47(4):450-9.

21. Jabak S, Vigneswaran T V., Charakida M, Kasapoglu T, Zidere V, Simpson JM, et al. Initial Experience of Superb Microvascular Imaging for Key Cardiac Views in Foetal Assessment before 15 Weeks Gestation. Fetal Diagn Ther. 2019;47(4):268-76.

22. Yeo L, Luewan S, Romero R. Fetal IntelligentNavigation Echocardiography (FINE)Detects98\% of Congenital Heart Disease. J Ultrasound Med. 2018;37(11):2577-93.

23. Yagel S, Cohen SM, Messing B. First and early second trimester fetal heart screening. Curr Opin Obstet Gynecol. 2007;19(2):183-90.

24. Yagel S, Cohen SM, Achiron R. Examination of the fetal heart by five short-axis views: a proposed screening method for comprehensive cardiac evaluation. Ultrasound Obstet Gynecol. 2001;17(5):367-9.

25. Harris GR, Church CC, Dalecki D, et al. Comparison of Thermal Safety Practice Guidelines for Diagnostic Ultrasound Exposures. Ultrasound Med Biol. 2016;42(2):345-357.

\section{TABLES}

Table I. Possible cardiac pathologies that can be detected by ultrasound in the first and second trimesters.

Table I. Possible cardiac pathologies that can be detected by ultrasound in the first and second trimesters.

Tricuspid, Mitral, Aortic, and Pulmonary Atresia

Hypoplastic Left Heart Syndrome

Complete and Congenitally Corrected Transposition of the Great Arteries

Double Outlet Right Ventricle

Common Arterial Trunk

Tetralogy of Fallot

Large Atrial and Ventricular Septal Defects

Complex Heart Defects Type Laterality

Table modified from Carvalho JS, 2004 (6).

Table II. Cardiac pathologies that cannot be detected by ultrasound in the first trimester and early second 
trimester.

Table II. Cardiac pathologies that cannot be detected by ultrasound in the first trimester and early second trimester.

Evolutionary injuries such as Mild Aortic / Pulmonary Stenosis, Mild Tricuspid and Mitral Valve Abnormalities, Coarctati Ventricular, Septal Primum, and Atrioventricular Septal defects by size

Fallot tetralogy with normal-sized Pulmonary Artery

Pulmonary Venous Return Abnormalities

Table modified from Carvalho JS, 2004 (6)

Table III. The sequence of Early Fetal Cardiac Assessment Sections.

Table III. The sequence of Early Fetal Cardiac Assessment Sections.

1. Abdomen and stomach

2. Four- chamber view

3. Left ventricular outflow tract

4. Right ventricular outflow tract

5. Three-vessel view and / or Three-vessel-trachea view

6. Ductal arch

7. Aortic arch

8. Both cava veins

Modified table from references $(22,24)$.

Figure 1. First-trimester fetal echocardiography of articles review flowchart.

Figure 2. Right sagittal view of the chest and fetal abdomen showing with color Doppler mapping, the cardiac silhouette, the aorta, the umbilical vein, and the venous ductus. A flow waveform in a normal venous ductus with a positive $\mathrm{A}$ wave (on the left) and a pathological one with an inverted $\mathrm{A}$ wave (on the right) is visualized. VU: umbilical vein. S: systole. D: diastole. A: atrial contraction. Source: Authors' own.

Figure 3. View of four apical cardiac chambers with pulsed Doppler at the level of the tricuspid valve. Tricuspid regurgitation is diagnosed if there is retrograde flow during ventricular systole with a velocity greater than $60 \mathrm{~cm} / \mathrm{s}$ and greater than half of the systole as evidenced in the figure on the right. On the left, normal flow is displayed at the level of the tricuspid valve. E: passive ventricular filling. A: active ventricular filling or atrial contraction.

Source: Authors' own.

Figure 4. Measurement of the fetal heart axis in a four-chamber lateral transverse section in an 8-week pregnancy (left), and an apical four-chamber transverse section in a 13-week pregnancy (right).

Source: Authors' own.

Figure 5. 1. Pulsed Doppler showing a monophasic wave in the atrioventricular flow at 8 weeks. 2. Pulsed Doppler at week 13, visualizing biphasic wave secondary to ventricular filling. E: passive ventricular filling. A: active ventricular filling or atrial contraction.

Source: Authors' own.

Figure 6. Sequential cuts of a fetal heart in the first and second trimester of gestation. 1. Situs solitus: transabdominal cut at the level of the gastric chamber and cut of four chambers. 2. Four-chamber section with atrioventricular flows. 3. Left ventricular outflow tract. Arrows point to the aorta. 4. Right ventricular outflow tract. Arrows point to the pulmonary artery. 5. 3-vessel cut. In the blue "V," there is the shapes of the crosses of the aortic and ductal arch (left), and outlined in yellow the three vessels (right). 6. Ductal 
arch outlined in yellow. 7. Aortic arch outlined in yellow. 8. The sagittal cut of the vena cava outlined in yellow. VU: umbilical vein. AO: aorta. CC: heart circumference. CT: chest circumference. AP: pulmonary artery. VCS: superior vena cava. 3VT: three vessels trachea.

Source: Authors' own.

Databases:

Embase: (5)

Medline: (12)

PubMed: (15)

ScienceDirect: (4)

Ovid: (2)

MD Consult: (4)

Clinical Key: (3)

Scielo: (2)
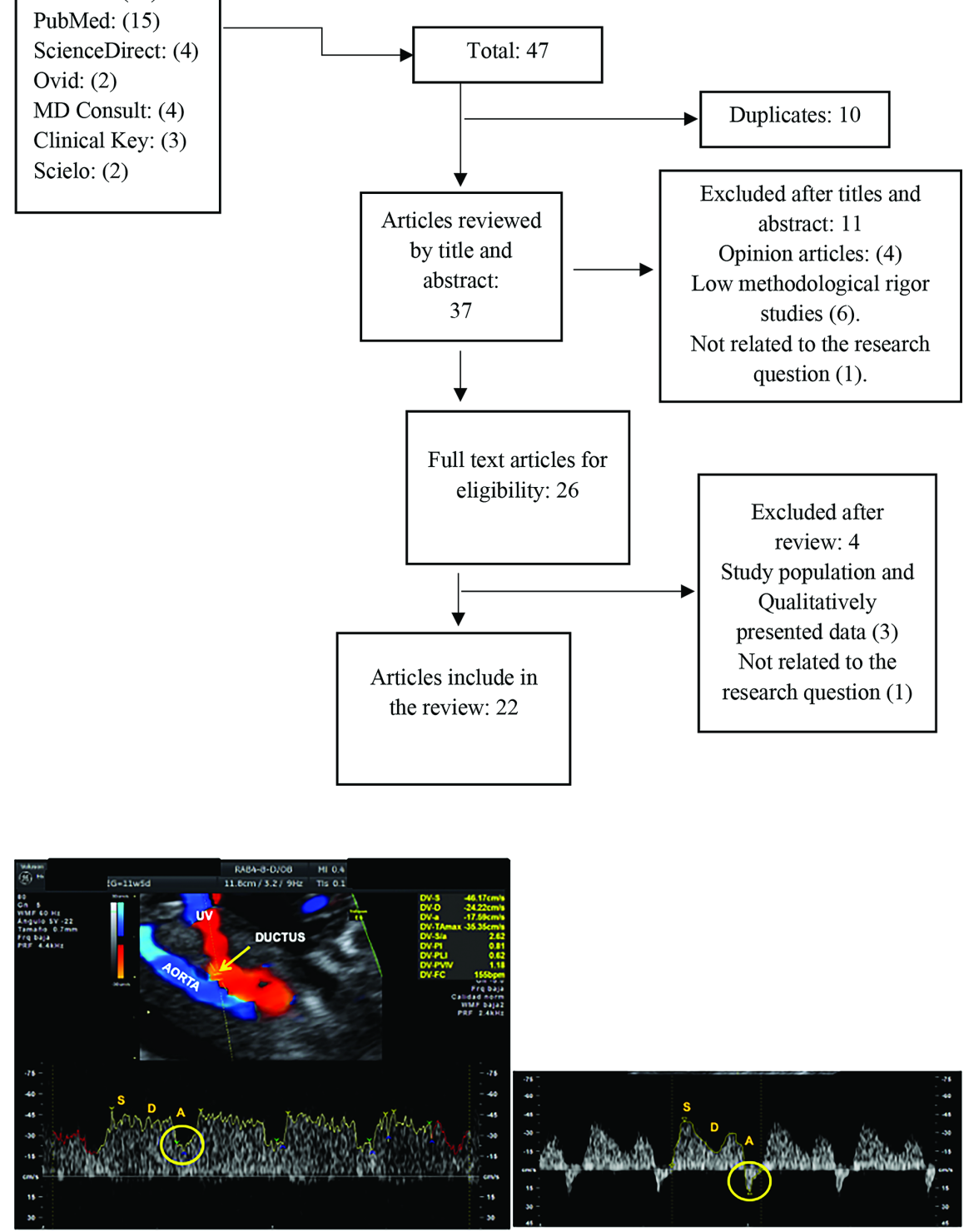

WAVE A NORMAL POSITIVE OF THE VENOUS DUCTUS

WAVE A PATHOLOGICAL REVERSE OF THE VENOUS DUCTUS 


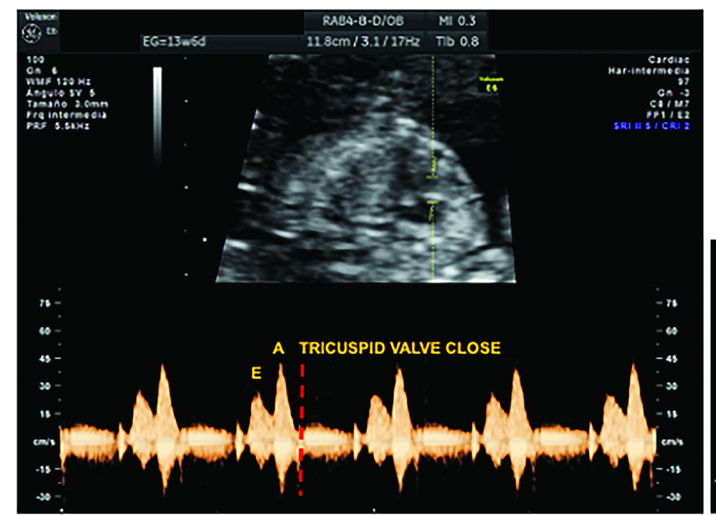

NORMAL TRICUSPID FLOW

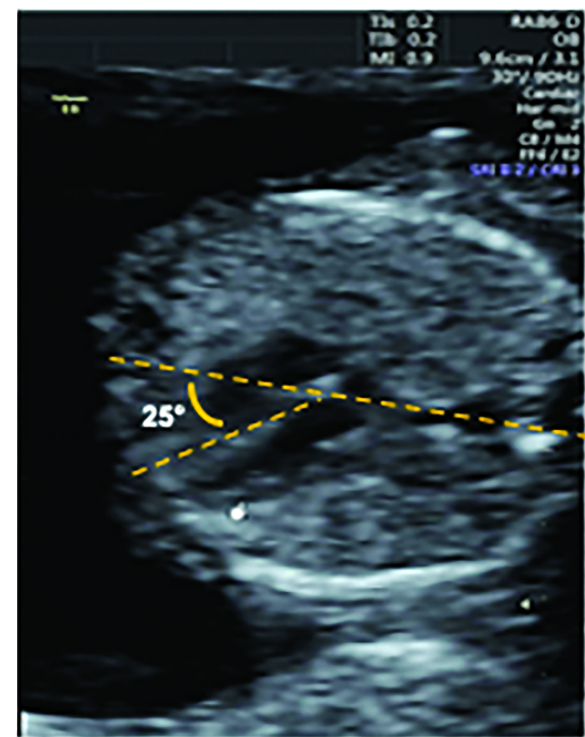

\section{CARDIAC AXIS}
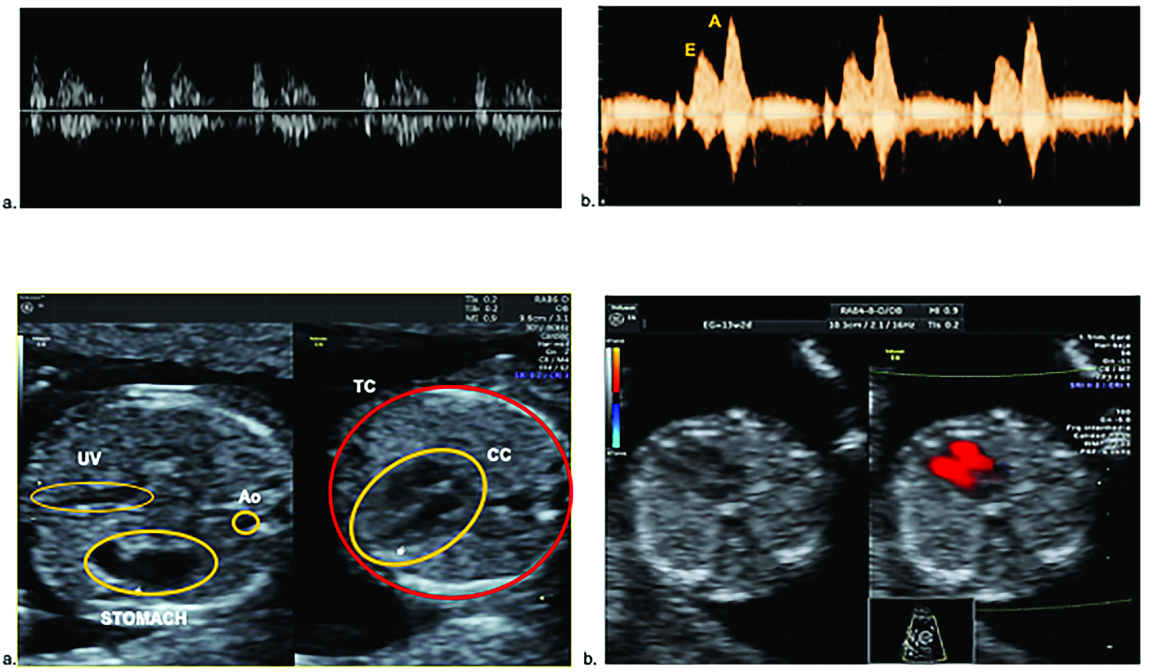

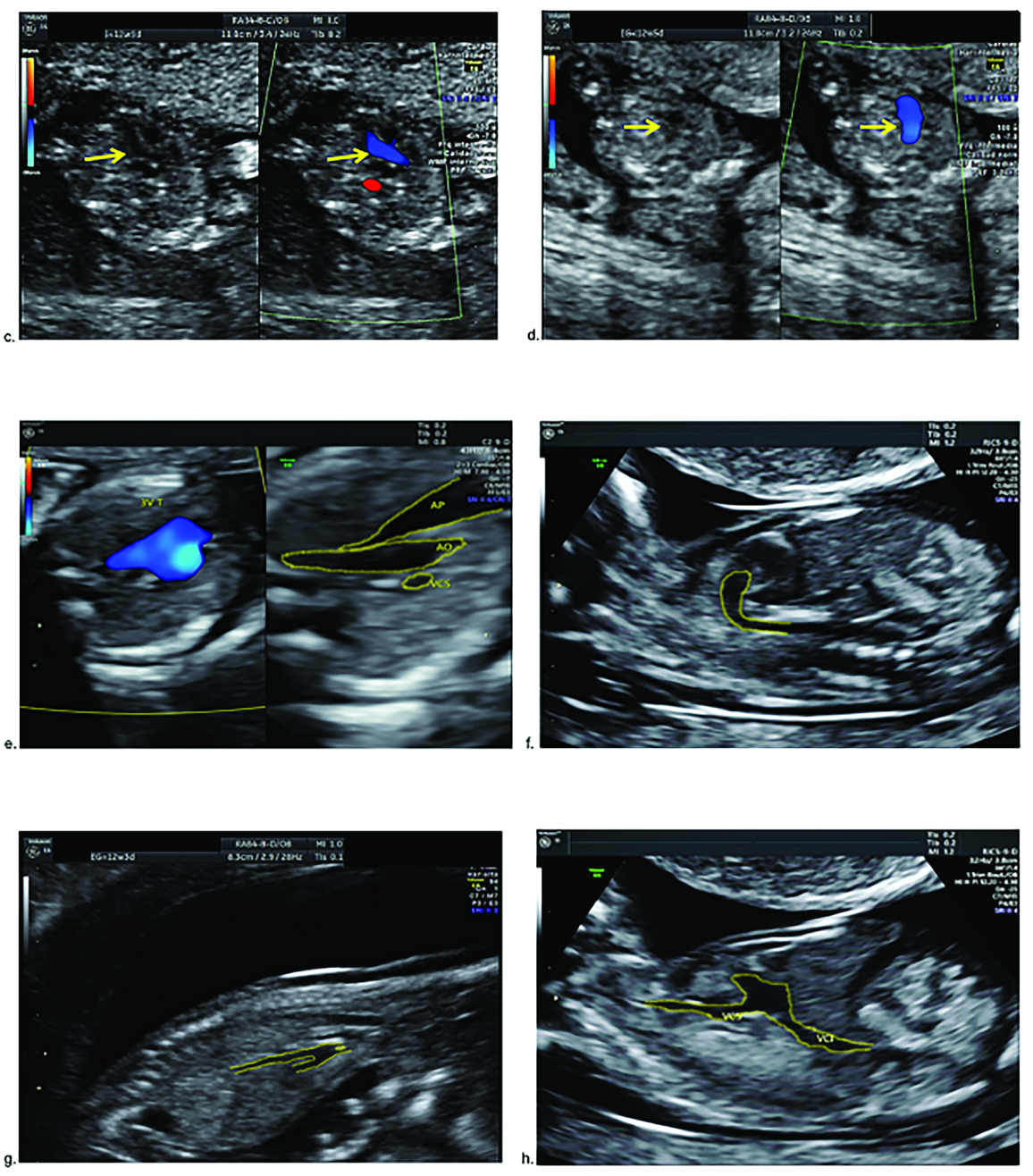